TRANSACTIONS OF THE

AMERICAN MATHEMATICAL SOCIETY

Volume 357, Number 9, Pages 3757-3774

S 0002-9947(05)03757-8

Article electronically published on March 31, 2005

\title{
LAGRANGIAN TORI IN HOMOTOPY ELLIPTIC SURFACES
}

\author{
TOLGA ETGÜ, DAVID MCKINNON, AND B. DOUG PARK
}

\begin{abstract}
Let $E(1)_{K}$ denote the symplectic four-manifold, homotopy equivalent to the rational elliptic surface, corresponding to a fibred knot $K$ in $S^{3}$ constructed by R. Fintushel and R. J. Stern in 1998. We construct a family of nullhomologous Lagrangian tori in $E(1)_{K}$ and prove that infinitely many of these tori have complements with mutually non-isomorphic fundamental groups if the Alexander polynomial of $K$ has some irreducible factor which does not divide $t^{n}-1$ for any positive integer $n$. We also show how these tori can be non-isotopically embedded as nullhomologous Lagrangian submanifolds in other symplectic 4-manifolds.
\end{abstract}

\section{INTRODUCTION}

In this paper, for each fibred knot $K$ in $S^{3}$, we give a new construction of an infinite family of nullhomologous Lagrangian tori in the symplectic four-manifold $E(1)_{K}=[E(1) \backslash \nu F] \cup\left[S^{1} \times\left(S^{3} \backslash \nu K\right)\right]$, homotopy equivalent to the rational elliptic surface $E(1)=\mathbb{C P}^{2} \# 9 \overline{\mathbb{C P}}^{2}$. $\quad(\nu F$ denotes a tubular neighborhood of a smooth torus fiber $F$ of the elliptic fibration.) After computing the fundamental groups of the complements of these nullhomologous tori, we are able to distinguish the isotopy types of infinitely many of them when the Alexander polynomial $\Delta_{K}(t)$ of $K$ satisfies a simple condition. Here is the exact statement:

Theorem 1. Let $K$ be a fibred knot in $S^{3}$. There are infinitely many nullhomologous non-isotopic Lagrangian tori in $E(1)_{K}$ whose complements have nonisomorphic fundamental groups, unless every irreducible factor of $\Delta_{K}(t)$ divides $t^{n}-1$ for a positive integer $n$, or equivalently, unless every root of $\Delta_{K}(t)$ is a root of unity.

In particular, if $\Delta_{K}(t)$ has an irreducible factor that has no root of absolute value 1 , then one can find non-isotopic nullhomologous Lagrangian tori in $E(1)_{K}$ (see Theorem 8). Note that the condition in the above theorem is satisfied by the figure-eight knot and its product with any other fibred knot, but not satisfied by torus knots. This is a consequence of the fact (see p. 326 of [20]) that the Alexander polynomial of a fibred knot is the characteristic polynomial of its monodromy map $\phi$, which could be finite order (periodic), reducible or pseudo-Anosov according to Thurston's classification theorem for surface diffeomorphisms in [22]. If $\phi$ is finite order, then its characteristic polynomial divides $t^{n}-1$ for some $n$. Note that the monodromy map of $(a, b)$ torus knot has order $|a b|$.

Received by the editors March 21, 2004.

2000 Mathematics Subject Classification. Primary 53D12, 57M05, 57R17; Secondary 57R52.

The second author was partially supported by an NSERC research grant.

The third author was partially supported by NSERC and CFI/OIT grants. 
In [8], the fundamental groups of the complements of the symplectic tori that were constructed in [4]-[7] are computed. Apparently, when $K$ is a fibred hyperbolic knot in $S^{3}$ it is possible to construct an infinite family of homologous symplectic tori which could be distinguished by the fundamental groups of their complements in $E(1)_{K}$. According to [23, the monodromy $\phi$ is pseudo-Anosov if and only if the knot $K$ is hyperbolic. We conjecture that the conclusion of Theorem 1 holds for all hyperbolic knots (see Example 14). The techniques used to distinguish the fundamental groups in $[8$ are quite different than the ones in this paper.

Knotted, i.e. homologous but not smoothly isotopic, Lagrangian tori in fourmanifolds were first constructed by Vidussi in 24] and then in a larger class of 4-manifolds by Fintushel and Stern in [11. They detected knottedness by (indirectly) computing the Seiberg-Witten invariants of the complements. In [1, Auckly distinguished the fundamental groups of the complements of such tori to prove nonisotopy. His argument applies only when the ambient manifold is $E(1)_{K \# \bar{K}}$, where $K$ is a fibred knot and $\bar{K}$ is the mirror of it. The non-isotopic Lagrangian tori we construct here are considerably simpler than the ones previously constructed, and can be embedded into a more general class of symplectic 4-manifolds that are not necessarily closed (see Theorem[17). Unfortunately, the techniques employed here and in the above-mentioned papers do not work when $K$ is the unknot. However, a different construction in 19 gives a family of homologous non-isotopic Lagrangian tori in $E(1)_{\text {unknot }}=E(1)$. More homologically essential family of Lagrangian tori will be constructed in [9].

In Section 2, we present a generalization of the Fintushel-Stern link surgery construction in [10. In Section 3 we give a simple construction of nullhomologous Lagrangian tori in $E(1)_{K}$. In Section 4 we compute the fundamental group of the complement of such a torus. Then we use Fox's free differential calculus to obtain the Alexander ideal of this group. A purely algebraic argument to distinguish these ideals (hence the groups) is given in Section 5. To our pleasant surprise, the argument dabbles into the realm of algebraic number theory. A brief discussion on a generalization to other symplectic 4-manifolds is in Section 6

\section{Generalized LINK SURGERY}

We present a generalization of the link surgery construction of Fintushel and Stern in [10] as follows. Let $L=\bigcup_{i=1}^{n} K_{i} \subset S^{3}$ be an $n$-component link, where $K_{i}$ is the $i$-th component. Let $\nu L=\bigcup_{i=1}^{n} \nu K_{i}$ denote its tubular neighborhood. The complement of $\nu L$ has boundary $\partial\left(S^{3} \backslash \nu L\right)=\bigcup_{i=1}^{n} \partial\left(\nu K_{i}\right) \cong \coprod_{i=1}^{n} T^{2}$, a disjoint union of $n$ copies of 2-torus. For each $i=1, \ldots, n$ let $X_{i}$ be a 4 -manifold with a boundary component $T_{i}^{3} \subset \partial X_{i}$, which is a 3 -torus. Let $\varphi_{i}: T_{i}^{3} \rightarrow S^{1} \times \partial\left(\nu K_{i}\right)$ be a diffeomorphism between 3 -tori.

Definition 2. The ordered collection $\mathfrak{D}=\left(\left\{X_{i}\right\}_{i=1}^{n} ;\left\{\varphi_{i}\right\}_{i=1}^{n}\right)$ is called a link surgery gluing data for an $n$-component link $L$. We define the link surgery manifold corresponding to $\mathfrak{D}$ to be the 4-manifold (possibly with boundary)

$$
L(\mathfrak{D})=\left[\coprod_{i=1}^{n} X_{i}\right] \cup_{\left\{\varphi_{i}\right\}}\left[S^{1} \times\left(S^{3} \backslash \nu L\right)\right]
$$

where we identify the $T_{i}^{3}$ boundary component of $X_{i}$ with the $i$-th component of the boundary of $S^{1} \times\left(S^{3} \backslash \nu L\right)$ via the gluing diffeomorphism $\varphi_{i}$. 
Homotopy rational elliptic surfaces. For our purposes we only need to look at a particular 2-component link $L$ which is a Hopf link in Figure1. Let us choose the following oriented factorizations of the boundary:

$$
\partial\left[S^{1} \times\left(S^{3} \backslash \nu L\right)\right] \cong\left[S^{1} \times \mu(A) \times \lambda(A)\right] \cup\left[S^{1} \times \lambda(B) \times \bar{\mu}(B)\right] .
$$

Here, $\mu(\cdot)$ and $\lambda(\cdot)$ denote the meridian and the longitude of a knot, respectively. A bar over $\mu$ means negative orientation. Note that both 3 -tori are given the boundary orientation coming from an orientation of the 4-manifold $S^{1} \times\left(S^{3} \backslash \nu L\right)$.

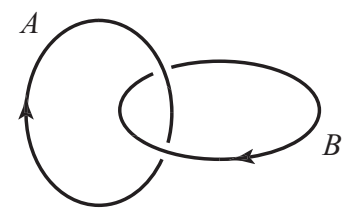

Figure 1. Hopf link $L=A \cup B$

Let $F$ denote a generic torus fiber of a rational elliptic surface $E(1) \cong \mathbb{C P}^{2} \# 9 \overline{\mathbb{C P}}^{2}$. Fix a cartesian product decomposition $F=\gamma_{1} \times \gamma_{2}$, where each $\gamma_{k} \cong S^{1}(k=1,2)$ is an embedded circle in $E(1)$. The elliptic fibration of $E(1)$ gives a canonical framing $\nu F \cong D^{2} \times \gamma_{1} \times \gamma_{2}$ and so

$$
\partial[E(1) \backslash \nu F] \cong T^{3} \cong \bar{\mu}(F) \times \gamma_{1} \times \gamma_{2},
$$

where $\mu(F)$ denotes the "meridian" of $F$ or the "rim circle" $\partial D^{2} \subset D^{2} \times \gamma_{1} \times \gamma_{2} \cong$ $\nu F$.

Let $M_{K}$ denote the 3-manifold that is the result of the 0-framed surgery on $S^{3}$ along $K$. Choose a meridian circle $\mu=\mu(K)$ in $M_{K}$. Define $T_{\mu}=S^{1} \times \mu \subset S^{1} \times M_{K}$. Note that $T_{\mu}$ has self-intersection 0 and there is a canonical framing of $T_{\mu}$ in $S^{1} \times M_{K}$ given by the minimal genus Seifert surface of the knot $K$. We shall always use this framing to trivialize

$$
\partial\left[\left(S^{1} \times M_{K}\right) \backslash \nu T_{\mu}\right]=\partial\left[S^{1} \times\left(S^{3} \backslash \nu K\right)\right] \cong S^{1} \times \mu(K) \times \lambda(K) .
$$

We will be working with the following two link surgery gluing data which differ only in $\varphi_{2}$ :

$$
\begin{aligned}
\mathfrak{D}_{1}= & \left(\left\{X_{1}=[E(1) \backslash \nu F], X_{2}=\left[\left(S^{1} \times M_{K}\right) \backslash \nu T_{\mu}\right]\right\} ;\right. \\
& \left.\left\{\varphi_{1}=\left(\begin{array}{ccc}
-1 & 0 & 0 \\
0 & 1 & 0 \\
0 & 0 & 1
\end{array}\right), \varphi_{2}=\left(\begin{array}{ccc}
0 & 0 & -1 \\
1 & 0 & 0 \\
0 & 1 & 0
\end{array}\right)\right\}\right), \\
\mathfrak{D}_{2}= & \left(\left\{X_{1}=[E(1) \backslash \nu F], X_{2}=\left[\left(S^{1} \times M_{K}\right) \backslash \nu T_{\mu}\right]\right\} ;\right. \\
& \left.\left\{\varphi_{1}=\left(\begin{array}{ccc}
-1 & 0 & 0 \\
0 & 1 & 0 \\
0 & 0 & 1
\end{array}\right), \varphi_{2}=\left(\begin{array}{ccc}
0 & 0 & -1 \\
0 & -1 & 0 \\
1 & 0 & 0
\end{array}\right)\right\}\right) .
\end{aligned}
$$

Here, we have expressed the gluing diffeomorphisms by matrices in $G L(3, \mathbb{Z})$ representing the induced linear maps $\left(\varphi_{i}\right)_{*}$ between the first homology groups of the boundary 3-tori with respect to the obvious ordered bases corresponding to the oriented factorizations (2.1), (2.2) and (2.3) chosen above.

Lemma 3. Let $L$ be the Hopf link in $S^{3}$. Let $\mathfrak{D}_{1}$ and $\mathfrak{D}_{2}$ be link surgery gluing data given by (2.4) and (2.5). Then $L\left(\mathfrak{D}_{1}\right)$ and $L\left(\mathfrak{D}_{2}\right)$ are both diffeomorphic to the fiber sum $E(1)_{K}=E(1) \#_{F=T_{\mu}}\left(S^{1} \times M_{K}\right)$. 
Proof. It is easy to see that the Hopf link exterior $S^{3} \backslash \nu L$ is diffeomorphic to $S^{1} \times \mathbb{A}$, where $\mathbb{A} \cong S^{1} \times[\epsilon, 1]$ is an annulus $(0<\epsilon<1)$. Hence we have

$$
\left[S_{x}^{1} \times\left(S^{3} \backslash \nu L\right)\right] \cong\left[S_{x}^{1} \times\left(S^{1} \times \mathbb{A}\right)\right] \cong T^{3} \times[\epsilon, 1],
$$

where we have used the notation $S_{x}^{1}$ to denote the first circle factor in $T^{3}$ to distinguish it from other $S^{1}$ factors. It follows that for both $j=1,2$,

$$
L\left(\mathfrak{D}_{j}\right)=[E(1) \backslash \nu F] \cup_{\varphi_{1}}\left[T^{3} \times[\epsilon, 1]\right] \cup_{\varphi_{2}}\left[\left(S^{1} \times M_{K}\right) \backslash \nu T_{\mu}\right] .
$$

Now $\varphi_{1}$ gives the boundary identification

$$
T^{3} \times\{1\}=S_{x}^{1} \times \mu(A) \times \lambda(A)=\mu(F) \times \overbrace{\gamma_{1} \times \gamma_{2}}^{F},
$$

while $\varphi_{2}$ in $\mathfrak{D}_{1}$ identifies factor-wise

$$
T^{3} \times\{\epsilon\}=S_{x}^{1} \times \lambda(B) \times \bar{\mu}(B)=\bar{\lambda}(K) \times \overbrace{S^{1} \times \mu(K)}^{T_{\mu}},
$$

and $\varphi_{2}$ in $\mathfrak{D}_{2}$ identifies factor-wise

$$
T^{3} \times\{\epsilon\}=S_{x}^{1} \times \lambda(B) \times \bar{\mu}(B)=\bar{\lambda}(K) \times \overbrace{\bar{\mu}(K) \times S^{1}}^{T_{\mu}} .
$$

For both $\mathfrak{D}_{1}$ and $\mathfrak{D}_{2}$, the fiber $F$ gets identified with $T_{\mu}$ and, after an orientation reversal, the meridian $\mu(F)$ gets identified with $\lambda(K)$, which is the meridian of $T_{\mu}$. It follows that

$$
L\left(\mathfrak{D}_{j}\right) \cong[E(1) \backslash \nu F] \cup_{\mu(F) \times F=\mu\left(T_{\mu}\right) \times T_{\mu}}\left[\left(S^{1} \times M_{K}\right) \backslash \nu T_{\mu}\right]=E(1)_{K}
$$

for both $j=1,2$. Finally, for an alternative proof, we remark that one can use the result of Matumoto in [18] that every orientation-preserving self-diffeomorphism of $\partial(\nu F) \cong T^{3}$ extends over $[E(1) \backslash \nu F]$. (Also see Theorem 8.3.11 in [14].)

\section{Construction of Lagrangian tori}

Our construction of nullhomologous Lagrangian tori is a modification of the construction in [6]. Using the diffeomorphism (2.6) in the proof of Lemma 3, we choose the symplectic form

$$
\omega_{0}=r d r \wedge d x+d y \wedge d \theta
$$

on $S_{x}^{1} \times\left(S^{3} \backslash \nu L\right)$, where $L=A \cup B$ is the Hopf link. Here, $x$ is the angular coordinate on $S_{x}^{1}, y$ is the angular coordinate parallel to $B$, and $(r, \theta)$ are the polar coordinates on a normal disk to $B$ with $\epsilon \leq r \leq 1$.

For each integer $q \geq 1$, draw a closed curve $C_{q}$ inside $\left(S^{3} \backslash \nu L\right)$ such that it is a $(1, q)$ torus knot lying on the boundary torus $\partial(\nu B)=\{(y, r, \theta) \mid r=\epsilon=$ constant $\}$. The linking numbers are $l k\left(C_{q}, A\right)=1$ and $l k\left(C_{q}, B\right)=q$. It follows immediately that $\left.d r\right|_{C_{q}}=0$. See Figure 2 which illustrates the case when $q=4$. Also see Figure 3 which shows the 3-component link $A \cup B \cup C_{q}$ after an isotopy.
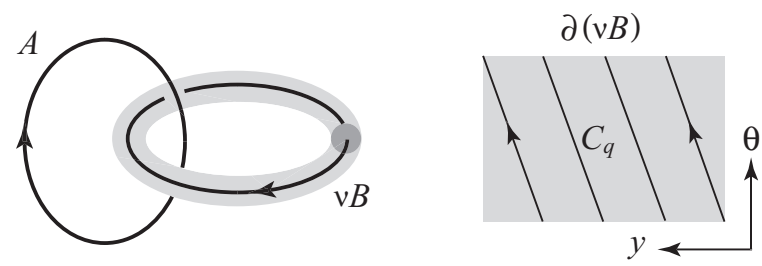

Figure 2. Closed curve $C_{q}$ on the torus $\partial(\nu B)$ 
For each integer $q \geq 1$, we define a torus $T_{q}=S_{x}^{1} \times C_{q} \subset\left[S_{x}^{1} \times\left(S^{3} \backslash \nu L\right)\right] \subset$ $L\left(\mathfrak{D}_{j}\right)=E(1)_{K}(j=1,2)$. We can easily compute that

$$
\left.\omega_{0}\right|_{T_{q}}=\left.(r d r \wedge d x)\right|_{S_{x}^{1} \times C_{q}}+\left.(d y \wedge d \theta)\right|_{S_{x}^{1} \times C_{q}}=0+0=0 .
$$

Lemma 4. Let $K$ be a fibred knot in $S^{3}$. For each pair of integers $j=1,2$ and $q \geq 1$, the torus $T_{q}=S_{x}^{1} \times C_{q}$ is a Lagrangian submanifold of $L\left(\mathfrak{D}_{j}\right) \cong E(1)_{K}$, and $\left[T_{q}\right]=0 \in H_{2}\left(E(1)_{K}\right)$.

Proof. In Section 2, we have built $E(1)_{K}$ using two "non-standard" link surgery identifications:

$$
E(1)_{K}=[E(1) \backslash \nu F] \cup_{\varphi_{1}}\left[S_{x}^{1} \times\left(S^{3} \backslash \nu L\right)\right] \cup_{\varphi_{2}}\left[S^{1} \times\left(S^{3} \backslash \nu K\right)\right] .
$$

Our argument below will not depend on $j$, i.e., it will go through regardless of which of the two link surgery gluing data is used. Recall that $F$ gets identified with the boundary torus $\partial(\nu A)$ in $\left(S^{3} \backslash \nu L\right)$ by $\varphi_{1}$, and $T_{\mu}=S^{1} \times \mu(K)$ gets identified with the boundary $\partial(\nu B)$ by $\varphi_{2}$. We can actually visualize these two tori in Figure 2 It follows that the volume forms of $F$ and $T_{\mu}$ are both identified (up to deformation) with the 2 -form $d y \wedge d \theta$.

We first need to show that the symplectic form $\omega_{0}$ on the middle piece can be extended to the whole of $E(1)_{K}$. When the knot $K$ is fibred, there is a fiber bundle

$$
S^{1} \times\left(S^{3} \backslash \nu K\right) \longrightarrow S^{1} \times \mu(K)=T_{\mu}
$$

with the fiber being the Seifert surface $\Sigma_{K}$ of $K$. Hence the 4-manifold $S^{1} \times\left(S^{3} \backslash \nu K\right)$ possesses a symplectic form $\omega_{2}$ that can be written locally as

$$
\omega_{2}=\Omega_{\Sigma_{K}}+\Omega_{T_{\mu}}
$$

where $\Omega$ denotes volume forms. Similarly, the elliptic fibration of $E(1)$ allows us to choose a symplectic form $\omega_{1}$ on $E(1) \backslash \nu F$ that can be written on the collar neighborhood of the boundary as

$$
\omega_{1}=\Omega_{S}+\Omega_{F},
$$

where $S$ is the image of a holomorphic section, minus a normal disk of $F$. We already saw that

$$
\varphi_{1}^{*}(d y \wedge d \theta)=\Omega_{F} \quad \text { and } \quad \varphi_{2}^{*}(d y \wedge d \theta)=\Omega_{T_{\mu}} .
$$

Now a punctured section $S$ and a Seifert surface $\Sigma_{K}$ can be glued together by an annulus in the middle:

$$
S_{x}^{1} \times\left\{y_{0}\right\} \times\left\{\theta_{0}\right\} \times[\epsilon, 1] \subset\left[T^{3} \times[\epsilon, 1]\right] \cong S_{x}^{1} \times\left(S^{3} \backslash \nu L\right),
$$

where $y_{0}$ and $\theta_{0}$ are constants. A volume form on this annulus is given by $r d r \wedge d x$. Thus $\Omega_{S}$ can be extended to $r d r \wedge d x$, which in turn can be extended to $\Omega_{\Sigma_{K}}$. So we have shown that the three symplectic forms $\omega_{0}, \omega_{1}$ and $\omega_{2}$ can be patched together. This, together with (3.1), proves that $T_{q}$ is a Lagrangian submanifold of $E(1)_{K}$.

Next we show that $T_{q}$ is nullhomologous in $E(1)_{K}$. Define $R_{1}=\mu(F) \times \gamma_{1}$ and $R_{2}=\mu(F) \times \gamma_{2}$, which are homologically essential tori in the boundary of $E(1) \backslash \nu F$. From the identification (2.7) we easily see that $\left[T_{q}\right]=\left[R_{1}\right]+q\left[R_{2}\right] \in H_{2}(E(1) \backslash \nu F)$. However, note that $\left[R_{1}\right]=\left[R_{2}\right]=0$ in $H_{2}\left(E(1)_{K}\right)$ since each $R_{k}$ gets identified (up to orientation) with either $\lambda(K) \times S^{1}$ or $\lambda(K) \times \mu(K)$, and so is bounded by either $\Sigma_{K} \times S^{1}$ or $\{\mathrm{pt}\} \times\left(S^{3} \backslash \nu K\right)$. 


\section{Fundamental group of the COMPlement}

When we view $E(1)_{K}$ as $L\left(\mathfrak{D}_{1}\right)$, i.e., when the boundary identification (2.8) $S^{1} \times \lambda(B) \times \bar{\mu}(B)=\bar{\lambda}(K) \times S^{1} \times \mu(K)$ is used, the fundamental group of $E(1)_{K} \backslash \nu T_{q}$, using the fact that the complement of a regular fiber in $E(1)$ is simply-connected, could be calculated as follows:

$$
\begin{aligned}
& \pi_{1}\left(E(1)_{K} \backslash \nu T_{q}\right) \\
& =\pi_{1}\left(E(1) \backslash \nu F \bigcup_{\mu(F) \times \gamma_{1} \times \gamma_{2}=S^{1} \times \mu(A) \times \lambda(A)} S^{1} \times\left(S^{3} \backslash \nu\left(A \cup B \cup C_{q}\right)\right)\right. \\
& \left.\bigcup_{S^{1} \times \lambda(B) \times \bar{\mu}(B)=\bar{\lambda}(K) \times S^{1} \times \mu(K)} S^{1} \times\left(S^{3} \backslash \nu K\right)\right) \\
& =\pi_{1}\left(\left(S^{1} \times\left(S^{3} \backslash \nu\left(B \cup C_{q}\right) \bigcup_{A=\partial D^{2}} D^{2}\right) \bigcup_{S^{1}=\partial D^{2}} D^{2}\right)\right. \\
& \left.\bigcup_{S^{1} \times \lambda(B) \times \bar{\mu}(B)=\bar{\lambda}(K) \times S^{1} \times \mu(K)} S^{1} \times\left(S^{3} \backslash \nu K\right)\right)
\end{aligned}
$$

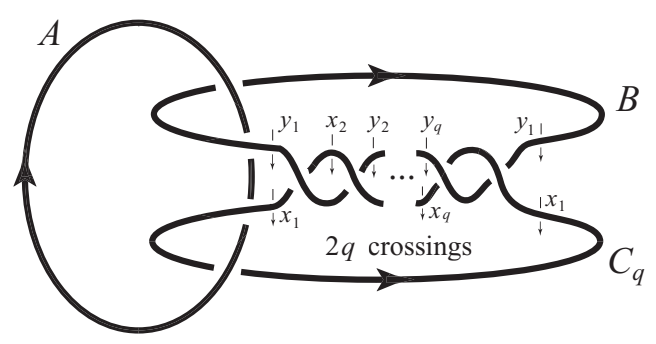

Figure 3. 3-component link $A \cup B \cup C_{q}$ in $S^{3}$

On the other hand, using the Wirtinger presentation of the link group of $B \cup C_{q}$ (see Figure 3), we can compute

$$
\begin{aligned}
& \pi_{1}\left(S^{3} \backslash \nu\left(B \cup C_{q}\right) \bigcup_{A=\partial D^{2}} D^{2}\right)=\pi_{1}\left(S^{3} \backslash \nu\left(B \cup C_{q}\right)\right) /\langle A\rangle \\
= & \left\langle x_{1}, \ldots, x_{q}, y_{1}, \ldots, y_{q} \mid y_{1} x_{1}=x_{2} y_{1}=y_{2} x_{2}=\cdots=y_{q} x_{q}=x_{1} y_{q}\right\rangle /\left\langle y_{1} x_{1}\right\rangle \\
= & \langle g \mid\rangle,
\end{aligned}
$$

where $g=x_{1}=\cdots=x_{q}=y_{1}^{-1}=\cdots=y_{q}^{-1}$, and in this group $\lambda(B)=x_{1} x_{2} \cdots x_{q}=$ $g^{q}$ and $\bar{\mu}(B)=y_{i}^{-1}=g$. Hence

$$
\pi_{1}\left(E(1)_{K} \backslash \nu T_{q}\right)=\frac{\langle g\rangle *\left[\pi_{1}\left(S^{1}\right) \oplus \pi_{1}\left(S^{3} \backslash \nu K\right)\right]}{g^{q}=S^{1}, 1=\lambda(K), g=\mu(K)} \cong \frac{\langle x\rangle \oplus \pi_{1}\left(S^{3} \backslash \nu K\right)}{\lambda(K)=1, \mu(K)^{q}=x},
$$

where $x$ is the generator of $\pi_{1}\left(S^{1}\right)$ which is identified with $g^{q}$.

In other words, to get the fundamental group of $E(1)_{K} \backslash \nu T_{q}$, we start with the knot group of $K$ and add two more relations. One of these relations kills the longitude and the other relation puts a specific power of the meridian in the center of the group. 
On the other hand, if we use the other boundary identification (2.9), namely $S^{1} \times \lambda(B) \times \bar{\mu}(B)=\bar{\lambda}(K) \times \bar{\mu}(K) \times S^{1}$, then a similar computation gives

$$
\pi_{1}\left(E(1)_{K} \backslash \nu T_{q}\right) \cong \frac{\langle x\rangle \oplus \pi_{1}\left(S^{3} \backslash \nu K\right)}{\lambda(K)=1, \mu(K)=x^{q}} .
$$

As demonstrated in the following example, the fundamental groups (4.2) of the complements we get by using the identification (2.9) seem to be totally independent of $q$, at least when the knot group has a presentation with only two generators. For this and other practical reasons, we will be concentrating on the group (4.1) which comes from the identification (2.8). After the following example we will focus only on the family of tori $\left\{T_{q}\right\}_{q \geq 1}$ in $L\left(\mathfrak{D}_{1}\right) \cong E(1)_{K}$. Apparently, in some cases, infinitely many of these Lagrangian tori cannot be distinguished by the fundamental groups of their complements either. Before considering the general case, we give a couple of examples to demonstrate the effect of the choice of knot $K$.

Example 5 (Torus knots). Let $K=\tau(a, b)$ be the $(a, b)$ torus knot. Then we have the following presentation of the knot group of $K$ (see e.g. page 47 in [3]):

$$
\left\langle u, v \mid u^{a}=v^{b}\right\rangle
$$

and moreover, in this presentation $\mu=u^{c} v^{d}$ and $\lambda=u^{a} \mu^{-a b}$ represent (for a suitably chosen base point) the meridian and the longitude, respectively, where $c$ and $d$ are relatively prime integers satisfying $a d+b c=1$. By using this presentation and (4.1) we obtain

$$
\begin{aligned}
\pi_{1}\left(E(1)_{K} \backslash \nu T_{q}\right) & =\frac{\langle x\rangle \oplus \pi_{1}\left(S^{3} \backslash \nu K\right)}{\lambda(K)=1, \mu(K)^{q}=x} \\
& =\left\langle x, u, v \mid[x, u]=[x, v]=1, u^{a}=v^{b}=\left(u^{c} v^{d}\right)^{a b},\left(u^{c} v^{d}\right)^{q}=x\right\rangle \\
& =\left\langle\mu, u, v \mid\left[\mu^{q}, u\right]=\left[\mu^{q}, v\right]=1, u^{a}=v^{b}=\mu^{a b}, \mu=u^{c} v^{d}\right\rangle \\
& =\left\langle\mu, u, v \mid\left[\mu^{p}, u\right]=\left[\mu^{p}, v\right]=1, u^{a}=v^{b}=\mu^{a b}, \mu=u^{c} v^{d}\right\rangle,
\end{aligned}
$$

where $p=\operatorname{gcd}(q, a b)$. The last line follows from the fact that, being a power of both $u$ and $v, \mu^{a b}$ commutes with $u$ and $v$ regardless of $q$. This calculation shows that, when a torus knot $K=\tau(a, b)$ is used, the number of non-isomorphic groups that can be obtained by varying $q$ is bounded by the number of positive divisors of $a b$ (see Example 16).

Note that, if we use (4.2) instead of (4.1), then we get

$$
\begin{aligned}
\pi_{1}\left(E(1)_{K} \backslash \nu T_{q}\right) & =\frac{\langle x\rangle \oplus \pi_{1}\left(S^{3} \backslash \nu K\right)}{\lambda(K)=1, \mu(K)=x^{q}} \\
& =\left\langle x, u, v \mid[x, u]=[x, v]=1, u^{a}=v^{b}=\left(u^{c} v^{d}\right)^{a b}, u^{c} v^{d}=x^{q}\right\rangle \\
& \cong \mathbb{Z}
\end{aligned}
$$

regardless of $q$. For the last isomorphism, note that $u^{c} v^{d}=x^{q}$ implies $u^{c}=x^{q} v^{-d}$, and this implies (since $x$ commutes with $v$ ) $u^{b c}=x^{q b} v^{-b d}$, hence (by $v^{b}=u^{a}$ ) $u^{b c}=x^{q b} u^{-a d}$, so $u=u^{b c+a d}=x^{q b}$. Similarly, one can show that $v=x^{q a}$.

Example 6 (Figure-eight knot). The figure-eight knot is the hyperbolic knot in $S^{3}$ with the least number of crossings. For the projection as in p. 58 of 20] with Wirtinger generators $y=x_{1}$ and $z=x_{3}$, the longitude can be written as a word in the Wirtinger generators via the algorithm outlined in Remark 3.13 of [3] (p. 39)

$$
\lambda(K)=\left[z, y^{-1}\right]\left[y, z^{-1}\right]=z y^{-1} z^{-1} y y z^{-1} y^{-1} z .
$$




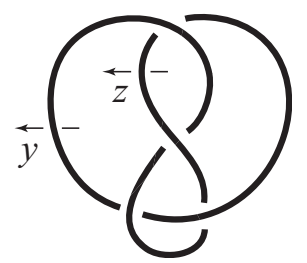

Figure 4. Figure-eight knot

By (4.1), the complement of $T_{q}$ has fundamental group

$$
\begin{gathered}
\pi_{1}\left(E(1)_{K} \backslash \nu T_{q}\right)=\langle x, y, z|[x, y]=[x, z]=1, y^{-1} z y z^{-1} y z y^{-1} z^{-1} y z^{-1}=1, \\
\left.z y^{-1} z^{-1} y y z^{-1} y^{-1} z=1, y^{q}=x\right\rangle \\
=\left\langle y, z \mid\left[y^{q}, z\right]=1, y^{-1} z y z^{-1} y z y^{-1} z^{-1} y z^{-1}=1, z y^{-1} z^{-1} y y z^{-1} y^{-1} z=1\right\rangle .
\end{gathered}
$$

It is clear that the abelianization of the above group is $\mathbb{Z}$. (The second relation becomes $y=z$, and the other two relations become trivial.) Now we apply Fox's free differential calculus as outlined in pp. 123-130 of [13]:

$$
\begin{aligned}
& \frac{\partial r_{1}}{\partial y}=1+y+y^{2}+\cdots+y^{q-1}-y^{q} z\left(y^{-1}+y^{-2}+\cdots+y^{-q}\right), \\
& \frac{\partial r_{1}}{\partial z}=y^{q}-1, \\
& \frac{\partial r_{2}}{\partial y}=-y^{-1}+y^{-1} z+y^{-1} z y z^{-1}-\left(z^{-1} y z^{-1}\right)^{-1}+\left(y z^{-1}\right)^{-1}, \\
& \frac{\partial r_{2}}{\partial z}=y^{-1}-y^{-1} z y z^{-1}+y^{-1} z y z^{-1} y-\left(y z^{-1}\right)^{-1}-1, \\
& \frac{\partial r_{3}}{\partial y}=-z y^{-1}+z y^{-1} z^{-1}+z y^{-1} z^{-1} y-z^{-1}, \\
& \frac{\partial r_{3}}{\partial z}=1-z y^{-1} z^{-1}-\left(y^{-1} z\right)^{-1}+z^{-1} .
\end{aligned}
$$

After passing to the Laurent polynomial ring $\mathbb{Z}[t, 1 / t]$, which is the group ring of the abelianization (i.e. we identify $y=z=t$ ), the Jacobian matrix becomes the following Alexander matrix:

$$
\begin{gathered}
{\left[\begin{array}{cc}
1-t^{q} & t^{q}-1 \\
-t^{-1}-t+3 & t^{-1}+t-3 \\
0 & 0
\end{array}\right] \sim\left[\begin{array}{cc}
1-t^{q} & t^{q}-1 \\
-t^{-1}-t+3 & t^{-1}+t-3
\end{array}\right]} \\
\sim\left[\begin{array}{cc}
1-t^{q} & t^{q}-1 \\
-t^{2}+3 t-1 & t^{2}-3 t+1
\end{array}\right] .
\end{gathered}
$$

The Alexander ideal (see p. 127 of [13]) $\mathcal{E}_{1}$ is the ideal in $\mathbb{Z}[t, 1 / t]$ that is generated by the entries of the above matrix and hence

$$
\mathcal{E}_{1}=\left(t^{q}-1, t^{2}-3 t+1\right)=\left(t^{q}-1, \Delta_{K}(t)\right) .
$$

As a consequence of Theorem 8 in Section 5 and as will be demonstrated in Example 10, we see that the Alexander ideal $\mathcal{E}_{1}$ is different for different values of $q$. Since $\mathcal{E}_{1}$ is an isomorphism invariant of a finitely presented group, this proves that the groups in $\left\{\pi_{1}\left(E(1)_{K} \backslash \nu T_{q}\right)\right\}_{q \geq 1}$ are mutually non-isomorphic. 
General case. Let $K$ be any fibred knot, let $\mathfrak{G}_{K}$ be the knot group with a Wirtinger presentation

$$
\mathfrak{G}_{K}=\left\langle x_{1}, \ldots, x_{n} \mid r_{1}, \ldots, r_{n-1}\right\rangle,
$$

and let $\mathfrak{G}_{K}^{q}$ be the group with the following presentation

$$
\mathfrak{G}_{K}^{q}=\left\langle x_{1}, \ldots, x_{n} \mid r_{1}^{\prime}, \ldots, r_{n-1}^{\prime}, r_{1}, \ldots, r_{n-1}\right\rangle,
$$

where $r_{i}^{\prime}=\left[x_{1}^{q}, x_{i+1}\right]$. It is clear that the abelianization of $\mathfrak{G}_{K}^{q}$ is $\mathbb{Z}$. Since the Alexander matrix class of a group obtained by using Fox calculus depends only on the group modulo its second commutator subgroup and the longitude of $K$ is in the second commutator subgroup of $\mathfrak{G}_{K}$, the Alexander ideal $\mathcal{E}_{1}\left(\pi_{1}\left(E(1)_{K} \backslash \nu T_{q}\right)\right.$ ), using the description of $\pi_{1}\left(E(1)_{K} \backslash \nu T_{q}\right)$ in (4.1), is the same as $\mathcal{E}_{1}\left(\mathfrak{G}_{K}^{q}\right)$. On the other hand, the Alexander matrix $A\left(\mathfrak{G}_{K}^{q}\right)$ of $\mathfrak{G}_{K}^{q}$ is

$$
\left[\begin{array}{ccccc}
1-t^{q} & t^{q}-1 & 0 & \cdots & 0 \\
1-t^{q} & 0 & t^{q}-1 & \cdots & 0 \\
\vdots & & & \ddots & \\
1-t^{q} & 0 & 0 & \cdots & t^{q}-1 \\
& & & & \\
& & & &
\end{array}\right] \sim\left[\begin{array}{ccccc}
0 & t^{q}-1 & 0 & \cdots & 0 \\
0 & 0 & t^{q}-1 & \cdots & 0 \\
\vdots & & & \ddots & \\
0 & 0 & 0 & \cdots & t^{q}-1 \\
& & & & \\
& & & &
\end{array}\right.
$$

where $A\left(\mathfrak{G}_{K}\right)$ and $A^{\prime}\left(\mathfrak{G}_{K}\right)$ are the Alexander matrices obtained by using two different presentations of $\mathfrak{G}_{K}$.

Remark 7 . Note that the equivalence of $(2 n-2) \times n$ matrices above is via column additions. In the notation of [12] (pp. 199-201), we are performing an elementary matrix transformation of type (III*) with $c_{2}=\cdots=c_{n}=1$, which correspond 1 to the Tietze transformation (II) of group presentations by adding $n-1$ new generators $y_{1}, \ldots, y_{n-1}$ satisfying $r_{j}^{\prime \prime}=y_{j} x_{1} x_{j+1}^{-1}=1$, followed by Tietze transformations (III),(0) and (II). Notice that $\left(x_{1} x_{j+1}^{-1}\right)^{\phi}=t t^{-1}=1$, and hence $\left(\partial r_{j}^{\prime \prime} / \partial x_{1}\right)^{\phi}=\left(y_{j}\right)^{\phi}=1=c_{j+1}$, where $\phi$ denotes the abelianization map. We also have $\left(\partial r_{j}^{\prime \prime} / \partial x_{j+1}\right)^{\phi}=\left(-y_{j} x_{1} x_{j+1}^{-1}\right)^{\phi}=-1$, and $\left(\partial r_{j}^{\prime \prime} / \partial y_{j}\right)^{\phi}=1$.

The Alexander ideal $\mathcal{E}_{1}\left(\mathfrak{G}_{K}^{q}\right)$ is generated by the determinants of all $(n-1) \times$ $(n-1)$ minors of $A\left(\mathfrak{G}_{K}^{q}\right)$. It is clear that $t^{q}-1$ divides all these determinants except for the ones which are the determinants of $(n-1) \times(n-1)$ minors of $A\left(\mathfrak{G}_{K}\right) \sim A^{\prime}\left(\mathfrak{G}_{K}\right)$. These latter determinants generate the Alexander ideal $\mathcal{E}_{1}\left(\mathfrak{G}_{K}\right)$ of the knot group which is always a principal ideal generated by the Alexander polynomial $\Delta_{K}$. Therefore

$$
\mathcal{E}_{1}\left(\mathfrak{G}_{K}^{q}\right)=\left(f_{1}, \ldots, f_{m}, \Delta_{K}\right)
$$

for some $f_{1}, \ldots, f_{m}$ which are all divisible by $t^{q}-1$. Thus, if we let $g_{q}(t)$ be the greatest common factor of $f_{1}, \ldots, f_{m}, \Delta_{K}$, then we can write

$$
\mathcal{E}_{1}\left(\mathfrak{G}_{K}^{q}\right)=\left(g_{q}(t)\right) J_{q},
$$

where $J_{q}$ is an ideal which is generated by relatively prime polynomials (over $\mathbb{Q}$ ) - in particular, the quotient ring $\mathbb{Z}[t, 1 / t] / J_{q}$ is finite. Moreover, if $\Delta_{K}(t)$ has an irreducible factor $f(t)$ which does not divide $t^{d}-1$ for any $d$, then $f(t)$ also

\footnotetext{
${ }^{1}$ In general, (III*) need not correspond to any Tietze transformation of group presentation.
} 
does not divide $f_{1}=\left(t^{q}-1\right)^{n-1}$, and hence does not divide $g_{q}(t)$. Therefore each generator of $J_{q}$ is divisible either by $f(t)$ (in the case of $\Delta_{K}(t)$ ) or by $\left(t^{q}-1\right) / g(t)$, where $g(t)$ is the greatest common factor of $g_{q}(t)$ and $t^{q}-1$. Thus, we obtain $J_{q} \subset\left(\left(t^{q}-1\right) / g(t), f(t)\right)$, so by Corollary [13 in Section [5] we conclude that there are infinitely many ideals of the form $\mathcal{E}_{1}\left(\mathfrak{G}_{K}^{q}\right)$, and hence Theorem 1 is proved.

\section{Algebraic Results And examples}

In this section we prove two algebraic theorems, Theorem 8 and Theorem 110 Theorem 11 is crucial in the proof of Theorem 1, which uses Corollary 13] Theorem 8 shows that if the hypotheses of Theorem 11 are strengthened slightly, then a much stronger conclusion can be drawn. Proposition 15] demonstrates that the condition on $\Delta_{K}(t)$ in Theorem 11 is almost a necessary one to distinguish the fundamental groups of the complements of infinitely many Lagrangian tori by using free calculus. The only polynomials $\Delta_{K}(t)$ that our methods cannot treat are those which do not divide $t^{n}-1$ for any $n$, yet every irreducible factor of $\Delta_{K}(t)$ is a divisor of some $t^{n}-1$.

Note that in this section, we will often refer to $\operatorname{gcd}\left(f_{1}, \ldots, f_{m}\right)$, where $f_{i} \in$ $\mathbb{Z}[t, 1 / t]$ are non-zero polynomials. By this we mean the monic polynomial $p(t)$ of largest degree, with the property that $p(t)$ divides $f_{i}$ for each $i$. If no such polynomial exists, we mean the gcd to be one, even if $1 \notin\left(f_{1}, \ldots, f_{m}\right)$. We apologize in advance for any confusion that this notation may cause.

Consider the ring $\mathbb{Z}[t, 1 / t]$ of Laurent polynomials in one variable with integer coefficients. Consider further the ideal $I_{q}=\left(t^{q}-1, \Delta_{K}(t)\right)$, where $q$ is a positive integer and $\Delta_{K}(t)$ is a monic polynomial in $t$ with integer coefficients with $\Delta_{K}(0)=$ 1. Note that the Alexander polynomial of a fibred knot will satisfy these conditions.

Theorem 8. Assume that $\Delta_{K}(t)$ is irreducible and has no roots of absolute value one. Then the quotient $\mathbb{Z}[t, 1 / t] / I_{q}$ is a finite ring of size $s_{q}$, and $s_{q} \rightarrow \infty$ as $q \rightarrow \infty$. Moreover, there is an integer $M$ such that for all $q, r>M$, we have $I_{q}=I_{r}$ if and only if $q=r$.

Proof. Let $t=\alpha$ be a root of $\Delta_{K}(t)$, and consider the quotient ring

$$
R=\mathbb{Z}[t, 1 / t] /\left(\Delta_{K}(t)\right) .
$$

Since $\Delta_{K}$ is irreducible, $R$ is isomorphic to the number ring $\mathbb{Z}[\alpha, 1 / \alpha]$, and the reduction of $I_{q}$ in $R$, denoted by $\bar{I}_{q}$, is the ideal generated by $\alpha^{q}-1$.

The field $\mathbb{Q}(\alpha)$ comes with a norm map to $\mathbb{Q}$, defined by

$$
N(\gamma)=\prod_{\sigma \in G} \sigma(\gamma)
$$

where $G=\operatorname{Gal}(F / \mathbb{Q})$ is the Galois group of the Galois closure $F$ of $\mathbb{Q}(\alpha) / \mathbb{Q}$. The norm map is clearly multiplicative, and has the property that $N(\gamma)=0$ if and only if $\gamma=0$. Moreover, if $x \in \mathbb{Z}[\alpha]$, then $N(x) \in \mathbb{Z}$, and $N(x)= \pm 1$ if and only if $x$ is a unit in $\mathbb{Z}[\alpha]$. We have $N(\alpha)= \pm \Delta_{K}(0)= \pm 1$, so $\alpha$ is a unit in $\mathbb{Z}[\alpha]$, and hence $\mathbb{Z}[\alpha, 1 / \alpha]=\mathbb{Z}[\alpha]$. (See [17] for proofs of these and other elementary facts about the norm map.)

Furthermore, the norm map provides a good estimate for the size $s_{q}$ of the quotient ring $R / \bar{I}_{q}$. Namely, there is a ring $T$, finitely generated as an $R$-module, such that for all $\gamma \in R$,

$$
|N(\gamma)|=\#(T / \gamma T) .
$$


(This depends on the fact that $\Delta_{K}(t)$ is monic, since this is precisely the case in which $\alpha$ is an algebraic integer. The ring $T$ is the integral closure of $R$ in its quotient field $\mathbb{Q}(\alpha)$.) Since $T$ is a finitely generated $R$-module, the cardinality of $T /\left(\alpha^{q}-1\right) T$ differs from the cardinality of $R / \bar{I}_{q}=R /\left(\alpha^{q}-1\right)$ by a multiplicative function bounded away from 0 and infinity. Thus, if we can show that $\left|N\left(\alpha^{q}-1\right)\right|$ grows exponentially with $q$, then we will have proven the theorem.

However, by the formula (5.1), we have

$$
\left|N\left(\alpha^{q}-1\right)\right|=\prod_{\sigma}\left|\sigma(\alpha)^{q}-1\right| .
$$

For each $\sigma$, if $|\sigma(\alpha)|<1$, then the corresponding factor in the product will go to 1 as $q \rightarrow \infty$. If $|\sigma(\alpha)|>1$, then the corresponding factor will grow exponentially with $q$.

We know that $\Delta_{K}$ has no roots with absolute value equal to 1 . Hence, for all $\sigma$, we have $|\sigma(\alpha)| \neq 1$. Since the product of these roots is a non-zero integer, it follows that there is some non-empty set of $\sigma$ for which $|\sigma(\alpha)|>1$, and hence that the value $\left|N\left(\alpha^{q}-1\right)\right|$ grows exponentially with $q$, as desired.

Remark 9. Computing the number $M$ is a matter of computing the index of $R=$ $\mathbb{Z}[\alpha]$ inside its ring of integers $T$, and of computing the absolute values of the roots of $\Delta_{K}$. If $\Delta_{K}$ is reducible, then the calculations in the proof of the theorem will grossly underestimate the actual size of $R / \bar{I}_{q}$, since in the proof we neglect the influence of factors of $\Delta_{K}$ other than $f(t)$.

Example 10 (Figure-eight knot). Let us examine the case of $\Delta_{K}(t)=t^{2}-3 t+1$ in detail. In this case, we can take

$$
\alpha=\frac{3+\sqrt{5}}{2} .
$$

The ring $\mathbb{Z}[\alpha]$ is equal to the $\operatorname{ring} \mathbb{Z}\left[\frac{1+\sqrt{5}}{2}\right]$, which is exactly equal to the ring of integers in the number field $\mathbb{Q}(\sqrt{5})$. Thus the norm of $\alpha^{q}-1$ gives the precise size of $\mathbb{Z}[\alpha] /\left(\alpha^{q}-1\right)$.

The field $\mathbb{Q}(\sqrt{5})$ has degree two over $\mathbb{Q}$, so it is a Galois extension whose Galois group has exactly two elements. The non-trivial automorphism of the field is the one which maps $a+b \sqrt{5}$ to $a-b \sqrt{5}$. It is clear that the absolute value of $\alpha$ is considerably larger than one, and that the absolute value of its conjugate $\frac{3-\sqrt{5}}{2}$ is less than one. Thus, as $q \rightarrow \infty$, we have

$$
\left|N\left(\alpha^{q}-1\right)\right| \sim\left(\frac{3+\sqrt{5}}{2}\right)^{q},
$$

so the ideals $I_{q}$ have exponentially larger quotients as $q \rightarrow \infty$. An examination of small values of $q$ quickly reveals that in fact, all the ideals $\bar{I}_{q}$ give different quotients of $R$, so that $M$ can be taken to be 0 .

If one removes the requirement that $\Delta_{K}$ has no roots of absolute value one, and replaces it with the requirement that $\Delta_{K}$ does not divide $t^{n}-1$ for any $n$, then one can prove a similar theorem to Theorem 8

Theorem 11. Let $\Delta_{K}(t)$ be a monic polynomial with $\Delta_{K}(0)=1$ and integer coefficients. Assume that $\Delta_{K}(t)$ does not divide $t^{n}-1$ for any $n$. Then the collection of ideals $I_{q}=\left(t^{q}-1, \Delta_{K}(t)\right)$ is infinite. 
Proof. Case one: $\Delta_{K}(t)$ has an irreducible factor $f(t)$ which does not divide $t^{n}-1$ for any $n$.

We first assume that $\Delta_{K}(t)=f(t)$. Let $\alpha$ be a root of $f(t)=0$. Then we have $\mathbb{Z}[t, 1 / t] / I_{q} \cong \mathbb{Z}[\alpha] /\left(\alpha^{q}-1\right)$, since $\alpha$ is a unit in the ring of algebraic integers. (The norm of $\alpha$ is \pm 1 because $f(0)=\Delta_{K}(0)=1$, and the units of $\mathbb{Z}[\alpha]$ are precisely the elements of norm \pm 1 .)

We need to show that $\mathbb{Z}[\alpha] /\left(\alpha^{q}-1\right)$ has unbounded size as $q \rightarrow \infty$. Since it may happen that $\Delta_{K}(t)$ has roots of absolute value one, the argument from the proof of Theorem 8 will not apply. Instead, we will show that for any prime number $p$, there is a value of $q$ for which $p$ divides $N\left(\alpha^{q}-1\right)$. By the same argument as in the proof of Theorem 8 , this will prove the theorem.

Thus, let $p$ be any prime, and consider the ring $R_{p}=\mathbb{Z}[\alpha] /(p)$. Since $\alpha$ is a unit, it follows that $\alpha$ is a unit in the ring $R_{p}$ as well. Hence there is some positive integer $M_{p}$ such that $\alpha^{M_{p}} \equiv 1(\bmod p)$. This means that if $q=M_{p}$, then $p$ divides $\alpha^{q}-1$, and therefore divides $N\left(\alpha^{q}-1\right)$. Since $\alpha$ is not a root of unity by hypothesis, it follows that $N\left(\alpha^{q}-1\right) \neq 0$, and hence must be at least size $p$. Since the set of primes is unbounded, it follows that $s_{q}=\#\left(\mathbb{Z}[t, 1 / t] / I_{q}\right)$ is also unbounded, and therefore that the set of ideals of the form $I_{q}$ is infinite.

If $\Delta_{K}(t) \neq f(t)$, then let $J_{q}=\left(t^{q}-1, f(t)\right)$. The set of ideals $\left\{J_{q}\right\}$ is an infinite set. Since $\Delta_{K}(t)$ only has a finite set of factors, it follows that there is an infinite set $S$ of natural numbers $q$ such that the set $\left\{J_{q} \mid q \in S\right\}$ is an infinite set, but $h(t)=\operatorname{gcd}\left(t^{q}-1, \Delta_{K}(t)\right)$ is independent of the choice of $q \in S$. Then for all $q \in S$, we can write $I_{q}=\left(t^{q}-1, \Delta_{K}(t)\right)=(h(t)) J_{q}^{\prime}$, where $J_{q}^{\prime}=\left(\left(t^{q}-1\right) / h(t), \Delta_{K}(t) / h(t)\right)$ is an ideal with $\mathbb{Z}[t, 1 / t] / J_{q}^{\prime}$ finite. Write $\Delta_{K}(t)=f(t) g(t) h(t)$; then

$$
J_{q}^{\prime}=\left(\left(t^{q}-1\right) / h(t), f(t) g(t)\right) \subset\left(\left(t^{q}-1\right) / h(t), f(t)\right) .
$$

To conclude the proof of case one, it suffices to show that the set of ideals of the form $H_{q}=\left(\left(t^{q}-1\right) / h(t), f(t)\right)$ for $q \in S$ is infinite.

It is clear that $\mathbb{Z}[t, 1 / t] / H_{q}$ is finite for all $q \in S$. Moreover, the cardinality of $\mathbb{Z}[t, 1 / t] / H_{q}$ is precisely the norm $N\left(\left(\alpha^{q}-1\right) / h(\alpha)\right)=N\left(\alpha^{q}-1\right) / N(h(\alpha))$ in $\mathbb{Q}(\alpha)$, where $\alpha$ is a root of $f(t)=0$. But $N(h(\alpha))$ is independent of $q$, and $N\left(\alpha^{q}-1\right)$ is unbounded, so we conclude that there are infinitely many ideals of the form $H_{q}$, and therefore of $J_{q}^{\prime}$ as well (since the size of $\mathbb{Z}[t, 1 / t] / H_{q}$ divides the size of $\mathbb{Z}[t, 1 / t] / J_{q}^{\prime}$ ). It follows immediately that the set $\left\{I_{q} \mid q \in S\right\}$ is infinite.

Case two: Every irreducible factor of $\Delta_{K}(t)$ is a divisor of $t^{n}-1$ for some $n$. If $\Delta_{K}(t)$ has no repeated roots, then in fact $\Delta_{K}(t)$ must divide $t^{n}-1$ for some $n$ : for each irreducible factor $f_{i}(t)$ of $\Delta_{K}(t)$, we have $f_{i}(t) \mid t^{n_{i}}-1$, so $\Delta_{K}(t)=\prod_{i} f_{i}(t)$ must divide $t \Pi n_{i}-1$.

Thus, we may assume that there is some irreducible factor $f(t)$ of $\Delta_{K}(t)$ for which $f(t)^{2}$ divides $\Delta_{K}(t)$ as well. In this case, it will happen that for sufficiently divisible $q$, the quotient $\mathbb{Z}[t, 1 / t] / I_{q}$ is not finite. As before, we assume first that $\Delta_{K}(t)=f(t)^{2}$. (It would suffice to assume that $\Delta_{K}(t)=f(t)$, of course, but sadly, the conclusion is false for $\Delta_{K}(t)=f(t)$; see Example [16.)

Thus, consider the set of ideals of the form $\left(t^{q}-1, f(t)^{2}\right)$, and let $n$ be an integer such that $f(t) \mid t^{n}-1$. If $q=k n$ for any positive integer $k$, then we have $f(t) \mid t^{q}-1$ 
as well, so we may write

$$
\begin{aligned}
\left(t^{q}-1, f(t)^{2}\right) & =(f(t))\left(\frac{t^{k n}-1}{f(t)}, f(t)\right) \\
& =(f(t))\left(p(t)\left(t^{(k-1) n}+t^{(k-2) n}+\ldots+1\right), f(t)\right),
\end{aligned}
$$

where $p(t)=\left(t^{n}-1\right) / f(t)$. Clearly, we may write $\left(t^{q}-1, f(t)^{2}\right)=(f(t)) J_{q}$, and it suffices to show that the following set of ideals is infinite:

$$
J_{q}=\left(p(t)\left(t^{(k-1) n}+t^{(k-2) n}+\cdots+1\right), f(t)\right) .
$$

Let $\alpha$ be a root of $f(t)=0$. Since $f(t)$ is an irreducible factor of $t^{n}-1$, it follows that $\alpha^{n}=1$, and that $\mathbb{Z}[t, 1 / t] / J_{q}$ is isomorphic to $\mathbb{Z}[\alpha] /(p(\alpha)(k))$. Since $t^{n}-1$ has no repeated roots, it follows that $p(\alpha) \neq 0$, and hence that the norm of $k p(\alpha)$ is an unbounded function of $k$. Since $q=k n$, it follows that there is an infinite set of ideals of the form $I_{q}$, as desired.

If $\Delta_{K}(t) \neq f(t)^{2}$, then we can use a similar argument to the one used at the end of the proof of Theorem 8 to show that the set of ideals of the form $I_{q}$ is infinite. Let $J_{q}$ be as above. We can find an infinite set $S$ of natural numbers such that every $q \in S$ is a multiple of $n$, the set $\left\{J_{q} \mid q \in S\right\}$ is infinite, but the polynomial $g(t)=\operatorname{gcd}\left(t^{q}-1, \Delta_{K}(t)\right)$ is independent of $q$. Then for all $q \in S$, we can write $I_{q}=\left(t^{q}-1, \Delta_{K}(t)\right)=(g(t)) J_{q}^{\prime}$, where $J_{q}^{\prime}=\left(\left(t^{q}-1\right) / g(t), \Delta_{K}(t) / g(t)\right)$ is an ideal with $\mathbb{Z}[t, 1 / t] / J_{q}^{\prime}$ finite. Since $q$ is divisible by $n$, it follows that $g(t)=f(t) h(t)$ for some polynomial $h(t)$. As before, it suffices to show that there are infinitely many ideals of the form $H_{q}=\left(\left(t^{q}-1\right) / g(t), f(t)\right) \supset J_{q}^{\prime}$.

But, as in Case one, we know that the size of $\mathbb{Z}[t, 1 / t] / H_{q}$ is just the norm $N\left(\left(\alpha^{q}-1\right) / g(\alpha)\right)$, where $\alpha$ is a root of the irreducible polynomial $f(t)$. Since $N\left(\left(\alpha^{q}-1\right) / g(\alpha)\right)=N\left(\left(\alpha^{q}-1\right) / f(\alpha)\right) / N(h(\alpha))$, and since $N\left(\left(\alpha^{q}-1\right) / f(\alpha)\right)$ is unbounded but $N(h(\alpha))$ is bounded, we conclude that there are infinitely many ideals of the form $H_{q}$, as desired.

Remark 12. Note that the hypothesis of Theorem 11] is strictly weaker than that of Theorem 8] since it is possible that $\Delta_{K}$ might be reducible, or have multiple roots (see Example 14). Moreover, there are even irreducible polynomials $\Delta_{K}$ for which Theorem 11 applies, but Theorem 8 does not. For example, the monic reciprocal integral polynomial $P(t)=t^{4}-t^{3}-t^{2}-t+1=\left(t^{2}-\left(\frac{1+\sqrt{13}}{2}\right) t+1\right)\left(t^{2}-\left(\frac{1-\sqrt{13}}{2}\right) t+1\right)$ satisfies $P(1)=-1$, hence there exists a fibred knot $K$ whose Alexander polynomial is $P(t)$, according to 2]. The maximum of two real roots of $P(t)$ is the smallest Salem number of degree 4. $P(t)$ is irreducible over $\mathbb{Q}$ and is not a divisor of $t^{n}-1$ for any $n$, but nevertheless has two roots of absolute value one (see 21]). The conclusion of Theorem 11] is only that the set of ideals of the form $I_{q}$ is infinite, which is also weaker than before. It is likely that the stronger conclusion of Theorem 8 holds in the more general setting, but the proof above does not show it.

Corollary 13. Let $\left\{E_{q}\right\}$ be a collection of ideals satisfying $E_{q} \subset\left(t^{q}-1, \Delta_{K}\right)$ for all $q$, where $\Delta_{K}$ is a monic integral polynomial with $\Delta_{K}(0)=1$. Assume that $\Delta_{K}(t)$ has some irreducible factor $f(t)$ which does not divide $t^{n}-1$ for any $n$. If for all $q$ we can write $E_{q}=\left(g_{q}(t)\right) J_{q}$ for some factor $g_{q}(t)$ of $\Delta_{K}(t)$ and some ideal $J_{q} \subset\left(\left(t^{q}-1\right) / \operatorname{gcd}\left(g_{q}(t), t^{q}-1\right), f(t)\right)$ with $\mathbb{Z}[t, 1 / t] / J_{q}$ finite, then there are infinitely many different ideals of the form $E_{q}$. 
Proof. It suffices to show that there are infinitely many ideals of the form $J_{q}$. It therefore further suffices to show that there are infinitely many ideals of the form

$$
H_{q}=\left(\left(t^{q}-1\right) / \operatorname{gcd}\left(g_{q}(t), t^{q}-1\right), f(t)\right) .
$$

By Theorem 11$]$ the ring $\mathbb{Z}[t, 1 / t] /\left(t^{q}-1, f(t)\right)$ is finite, and of unbounded size $s_{q}$. Since there are only finitely many possible factors $g_{q}(t)$ of $\Delta_{K}(t)$, there is some factor $g(t)$ such that there are infinitely many $q$ with $\operatorname{gcd}\left(g_{q}, t^{q}-1\right)=g$ and $s_{q}$ arbitrarily large. Moreover, since $f(t)$ is irreducible, for each such $q$, we have $s_{q}=N\left(\alpha^{q}-1\right)$, where $\alpha$ is a root of $f(t)$.

On the other hand, the size of $\mathbb{Z}[t, 1 / t] / H_{q}$ is equal to

$$
N\left(\left(\alpha^{q}-1\right) / g(\alpha)\right)=s_{q} / N(g(\alpha)) .
$$

Since $N(g(\alpha))$ is independent of $q$, it follows that the size of $\mathbb{Z}[t, 1 / t] / H_{q}$ is unbounded, and therefore that there are infinitely many ideals of the form $H_{q}$. The corollary follows.

We would like to include all hyperbolic fibred knots in the list of knots for which there is a corresponding homotopy elliptic surface with infinitely many non-isotopic nullhomologous Lagrangian tori. However, as demonstrated in the following example, knot $8_{20}$ (according to Rolfsen's list in [20]) has an Alexander polynomial which satisfies the condition in Theorem 11, but does not satisfy the conditions of Corollary 13. Note that $8_{20}$ is indeed hyperbolic (see [15) and fibred. (It has been checked in [16] that the prime knots with 10 or fewer crossings are fibred if and only if $\Delta_{K}(0)= \pm 1$. Alternatively, see Exer. 5.5 on p. 78 of [3].)

Example $14\left(\right.$ Knot $\left.8_{20}\right)$. To illustrate the case when the quotient ring $\mathbb{Z}[t, 1 / t] / I_{q}$ need not be finite, we will consider the case of $K=8_{20}$, whose Alexander polynomial i. $2 \Delta_{K}(t)=\left(t^{2}-t+1\right)^{2}$. Since $t^{2}-t+1$ divides $t^{6}-1$, it follows that $\Delta_{K}(t)$ does not divide $t^{n}-1$ for any $n$ (since $t^{n}-1$ has no repeated factors), but $\Delta_{K}(t)$ does not have any irreducible factor which does not divide $t^{n}-1$ for any $n$. Thus, we are not able to use Corollary 13 directly, but additional calculation will enable us to conclude that there are indeed infinitely many ideals of the form $\mathcal{E}_{1}\left(\mathfrak{G}_{K}^{q}\right)$.

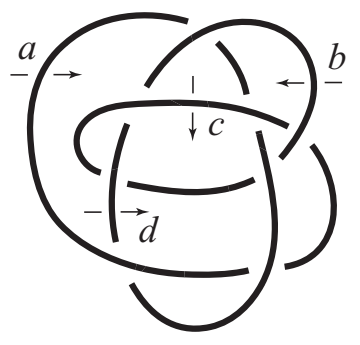

FiguRE 5. Knot $8_{20}$

In particular, the knot group for $K=8_{20}$ can be presented as

$$
\mathfrak{G}_{K}=\left\langle a, b, c, d \mid b^{-1} a d a^{-1} d^{-1} c d a d^{-1} a^{-1}, c^{-1} b^{-1} a d a d^{-1} a^{-1} b, d^{-1} c^{-1} b c\right\rangle,
$$

\footnotetext{
${ }^{2}$ There is a typo in the knot table (p. 336) in [3] where the Alexander polynomial is written as $t^{2}-t+1$.
} 
where the generators are as in Figure 5 Using Fox calculus, we compute that

$$
A\left(\mathfrak{G}_{K}\right) \sim\left[\begin{array}{cccc}
t^{-1}-2+t & -t^{-1} & t^{-1} & -t^{-1}+2-t \\
t^{-2}-t^{-1}+1 & -t^{-2}+t^{-1} & -t^{-1} & t^{-1}-1 \\
0 & t^{-2} & -t^{-2}+t^{-1} & -t^{-1}
\end{array}\right] .
$$

Hence the matrix $A\left(\mathfrak{G}_{K}^{q}\right)$ associated to $8_{20}$ is as follows:

$$
\left[\begin{array}{cccc}
1-t^{q} & t^{q}-1 & 0 & 0 \\
1-t^{q} & 0 & t^{q}-1 & 0 \\
1-t^{q} & 0 & 0 & t^{q}-1 \\
1-2 t+t^{2} & -1 & 1 & -1+2 t-t^{2} \\
1-t+t^{2} & -1+t & -t & t-t^{2} \\
0 & 1 & -1+t & -t
\end{array}\right] \sim\left[\begin{array}{cccc}
0 & t^{q}-1 & 0 & 0 \\
0 & 0 & t^{q}-1 & 0 \\
0 & 0 & 0 & t^{q}-1 \\
0 & -1 & 1 & -1+2 t-t^{2} \\
0 & -1+t & -t & t-t^{2} \\
0 & 1 & -1+t & -t
\end{array}\right]
$$

Note that the determinant of the $3 \times 3$ minor corresponding to rows $3,4,5$ and the last three columns is $t^{q}-1$. Thus the ideal $\mathcal{E}_{1}\left(\mathfrak{G}_{K}^{q}\right)$, generated by the determinants of all $3 \times 3$ minors of $A\left(\mathfrak{G}_{K}^{q}\right)$, is precisely the ideal $I_{q}=\left(t^{q}-1, \Delta_{K}(t)\right)$, which by Theorem 11 ranges over an infinite set of ideals.

Let us examine this in more detail. It turns out that for infinitely many $q$, the ring $\mathbb{Z}[t, 1 / t] / I_{q}$ is infinite. In particular, if $q=6 n$ is a multiple of 6 , then we have

$$
\begin{aligned}
I_{q} & =\left(t^{q}-1,\left(t^{2}-t+1\right)^{2}\right) \\
& =\left(t^{2}-t+1\right)\left(\left(t^{4}+t^{3}-t-1\right)\left(t^{6(n-1)}+\cdots+t^{6}+1\right), t^{2}-t+1\right) .
\end{aligned}
$$

Since this ideal is contained in the ideal $\left(t^{2}-t+1\right)$, we have an inclusion

$$
\mathbb{Z}[t, 1 / t] /\left(t^{2}-t+1\right) \hookrightarrow \mathbb{Z}[t, 1 / t] / I_{q}
$$

provided, of course, that $q$ is a multiple of 6 . Since the first of these rings is infinite, it follows that the other is, too.

However, following the proof (Case two) of Theorem 11 we can still show that there are infinitely many ideals of the form $I_{q}$ by showing that there are infinitely many ideals of the form $J_{n}=\left(\left(t^{4}+t^{3}-t-1\right)\left(t^{6(n-1)}+\cdots+t^{6}+1\right), t^{2}-t+1\right)$. Since $\mathbb{Z}[t, 1 / t] / J_{n}$ is a finite ring for all $n$, we can compute the cardinality of $\mathbb{Z}[t, 1 / t] / J_{n}$, and show that it is an unbounded function of $n$, and hence that there are infinitely many different ideals of the form $J_{n}$, and hence infinitely many ideals of the form $I_{q}$.

As before, we know that $\mathbb{Z}[t, 1 / t] / J_{n}$ is isomorphic to $\mathbb{Z}[\gamma] /(p(\gamma))$, where $p(t)=$ $\left(t^{4}+t^{3}-t-1\right)\left(t^{6(n-1)}+\cdots+t^{6}+1\right)$ and $\gamma$ is a primitive sixth root of unity (that is, $\gamma$ is a root of $\left.t^{2}-t+1=0\right)$. In particular, $\gamma^{6}=1$, and

$$
t^{4}+t^{3}-t-1=\frac{t^{6}-1}{t^{2}-t+1}
$$

so since $t^{6}-1$ has no repeated factors, it follows that

$$
\gamma^{4}+\gamma^{3}-\gamma-1 \neq 0
$$

Hence we obtain $p(\gamma)=C n$, where $C$ is some non-zero constant, independent of $n$. Thus, the cardinality of $\mathbb{Z}[t, 1 / t] / J_{n}$ is equal to the norm $N(p(\gamma))=|C|^{2} n^{2}$, which is obviously an unbounded function of $n$. We conclude that there are infinitely many ideals of the form $I_{q}=\mathcal{E}_{1}\left(\mathfrak{G}_{K}^{q}\right)$, as desired. 
An argument similar to that of Example 14 should work for many if not all hyperbolic fibred knots which satisfy the conditions of Theorem [1] but not of Corollary 13, but we cannot prove it at this time. However, we can show that if $\Delta_{K}(t)$ does not satisfy the hypothesis of Theorem 11 then our techniques are highly unlikely to work.

Proposition 15. If $\Delta_{K}$ divides $t^{n}-1$ for some $n$, then the set of ideals of the form $I_{q}=\left(t^{q}-1, \Delta_{K}\right)$ is a finite set.

Proof. Since $\Delta_{K}(t)$ is a factor of $t^{n}-1$, we have $t^{n}-1 \in I_{q}$. But $t^{q}-1 \in I_{q}$, too, so $\operatorname{gcd}\left(t^{n}-1, t^{q}-1\right)=t^{g}-1 \in I_{q}$, where $g=\operatorname{gcd}(n, q)$. In fact, we obtain $I_{q}=\left(t^{g}-1, \Delta_{K}\right)$, since $t^{g}-1$ divides $t^{q}-1$. But since $n$ is fixed, $g$ varies over the finite set of divisors of $n$, and hence there are only finitely many different ideals $I_{q}$, as desired.

Example 16 (Trefoils $\tau(3, \pm 2)$ ). Let us consider the rings

$$
R_{q}=\mathbb{Z}[t, 1 / t] /\left(t^{q}-1, t^{2}-t+1\right) .
$$

Since $t^{2}-t+1$ is a divisor of $t^{6}-1$, Theorem 11 does not apply. Indeed, the set of rings $R_{q}$ is finite, because the set of ideals $\left(t^{q}-1, t^{2}-t+1\right)$ is also a finite set.

To prove this, note that $R_{q}$ is isomorphic to the ring $\mathbb{Z}[\gamma] /\left(\gamma^{q}-1\right)$, where $\gamma=$ $e^{2 \pi i / 6}$ is a primitive sixth root of unity. Thus, immediately we can see that there are at most six of these ideals, corresponding to $q=1,2,3,4,5,6$. Furthermore, since $1 / \gamma=\gamma^{5}=1-\gamma$, it follows that $R_{1}$ is isomorphic to $R_{5}$ and $R_{2}$ is isomorphic to $R_{4}$, and the corresponding ideals are the same as well. Thus, we may restrict our attention to the rings $R_{1}, R_{2}, R_{3}$ and $R_{6}$. It is further useful to note that $\mathbb{Z}[\gamma]$ is equal to the ring of integers in the field $\mathbb{Q}(\gamma)$, so that in particular, $R_{q}$ has cardinality exactly equal to $N\left(\gamma^{q}-1\right)$.

Clearly, the ring $R_{6}$ is isomorphic to $\mathbb{Z}[\gamma]$, since $\gamma^{6}-1=0$. The other three rings are all finite, of different orders:

$R_{1}$ is the trivial ring, since $\gamma-1$ is a unit in $\mathbb{Z}[\gamma]$, with inverse $-\gamma . R_{2}$ is the field with three elements, because $\left(\gamma^{2}-1\right)$ is the same ideal as $(\gamma+1)$, and $N(\gamma+1)=3$.

$R_{3}$ is the field with four elements. We have

$$
\begin{aligned}
N\left(\gamma^{3}-1\right) & =N(\gamma-1) N\left(\gamma^{2}+\gamma+1\right) \\
& =\left(\gamma^{2}+\gamma+1\right)\left(\gamma^{-2}+\gamma^{-1}+1\right) \\
& =1+\gamma+\gamma^{2}+\gamma^{-1}+1+\gamma+\gamma^{-2}+\gamma^{-1}+1 \\
& =3+2\left(\gamma+\gamma^{-1}\right)+\left(\gamma^{2}+\gamma^{-2}\right) \\
& =3+2-1=4
\end{aligned}
$$

(Recall that $\gamma=e^{2 \pi i / 6}=\frac{1+\sqrt{-3}}{2}$.) This means that $R_{3}$ has four elements, so all that remains is to verify that it is a field. This is easily done: its non-zero elements can be represented by $1, \gamma$ and $1 / \gamma$, each of which has a multiplicative inverse.

\section{Generalization to other symplectic 4-Manifolds}

Our result generalizes readily to $E(n)_{K}(n>1)$ and more general link surgery manifolds as follows.

Theorem 17. Let $K$ be a fibred knot in $S^{3}$ whose Alexander polynomial $\Delta_{K}(t)$ has an irreducible factor none of whose roots is a root of unity. Let $X$ be a symplectic 
4-manifold (not necessarily closed) with a symplectic torus submanifold $T$ of selfintersection 0. If $\pi_{1}(X \backslash \nu T)=1$, then there are infinitely many nullhomologous non-isotopic Lagrangian tori in $X_{K}$. Here, $X_{K}=L\left(\mathfrak{D}_{1}^{\prime}\right)$, where $L$ is the Hopf link and the link surgery gluing data $\mathfrak{D}_{1}^{\prime}$ differs from $\mathfrak{D}_{1}$ in (2.4) only in that $X_{1}=X \backslash \nu T$.

Proof. The computation of fundamental groups $\pi_{1}\left(X_{K} \backslash \nu T_{q}\right)$ can be carried out in exactly the same way as before and yields the same result.

Finally we conjecture that the conclusion of Theorem [17 holds even when $K$ is any non-trivial fibred knot. One might be able to distinguish the isotopy types of $T_{q}$ via the framing invariants of Fintushel and Stern in [11. We plan to compute the framing invariants of $T_{q}$ and other families of Lagrangian tori in [9].

\section{ACKNOWLEDGMENTS}

We thank Hans U. Boden, Ronald Fintushel, Louis H. Kauffman, John Lawrence, Jason Lucier, John D. McCarthy, Andrew Nicas and Stefano Vidussi for helpful comments and stimulating discussions. The figures were produced by the third author using Adobe ${ }^{\circledR}$ Illustrator ${ }^{\circledR}$ Version 10.

\section{REFERENCES}

[1] D. Auckly: Topologically knotted Lagrangians in simply connected four manifolds, Proc. Amer. Math. Soc. 133 (2005), 885-889.

[2] G. Burde: Alexanderpolynome Neuwirthscher Knoten, Topology 5 (1966), 321-330. MR.0199858 (33:7998)

[3] G. Burde and H. Zieschang: Knots. Second edition. de Gruyter Studies in Mathematics, 5. Walter de Gruyter \& Co., Berlin, 2003. MR 1959408 (2003m:57005)

[4] T. Etgü and B. D. Park: Non-isotopic symplectic tori in the same homology class, Trans. Amer. Math. Soc. 356 (2004), 3739-3750. MR2055752

[5] _ : Homologous non-isotopic symplectic tori in a K3-surface, Commun. Contemp. Math. (to appear), math.GT/0305201.

[6] _ : Homologous non-isotopic symplectic tori in homotopy rational elliptic surfaces, Math. Proc. Cambridge Philos. Soc. (to appear), math.GT/0307029.

[7] _ : Symplectic tori in rational elliptic surfaces, math.GT/0308276.

[8] _ : A note on fundamental groups of symplectic torus complements in 4-manifolds, preprint.

[9] _ Homologous non-isotopic Lagrangian tori in symplectic 4-manifolds, in preparation.

[10] R. Fintushel and R. J. Stern: Knots, links and 4-manifolds, Invent. Math. 134 (1998), 363400. MR 1650308 (99j:57033)

[11] _ : Invariants for Lagrangian tori, Geom. Topol. 8 (2004), 947-968. MR2087074

[12] R. H. Fox: Free differential calculus. II. The isomorphism problem of groups. Ann. of Math. 59 (1954), 196-210. MR0062125 (15:931e)

[13] _ : A quick trip through knot theory, in Topology of 3-Manifolds and Related Topics (Proc. The Univ. of Georgia Institute, 1961), pp. 120-167, Prentice-Hall, Englewood Cliffs, 1962. MR0140099 (25:3522)

[14] R. E. Gompf and A. I. Stipsicz: 4-Manifolds and Kirby Calculus, Graduate Studies in Mathematics 20, Amer. Math. Soc., Providence, 1999. MF1707327 (2000h:57038)

[15] J. Hoste, M. Thistlethwaite and J. Weeks: The first 1, 701,936 knots, Math. Intelligencer 20 (1998), 33-48. MR.1646740 (99i:57015)

[16] T. Kanenobu: The augmentation subgroup of a pretzel link, Math. Sem. Notes Kobe Univ. 7 (1979), 363-384. MR 0557309 (81a:57008)

[17] D. A. Marcus: Number Fields. Universitext. Springer-Verlag, New York-Heidelberg, 1977. MR $0457396(56: 15601)$

[18] T. Matumoto: Extension problem of diffeomorphisms of a 3-torus over some 4-manifolds, Hiroshima Math. J. 14 (1984), 189-201. MR0750396 (86b:57016) 
[19] B. D. Park and S. Vidussi: Non-isotopic Lagrangian tori in elliptic surfaces, preprint.

[20] D. Rolfsen: Knots and Links. Second printing, with corrections. Mathematics Lecture Series, 7. Publish or Perish Inc., Houston, 1990. MF1277811 (95c:57018)

[21] C. L. Stewart: Private communication.

[22] W. P. Thurston: On the geometry and dynamics of diffeomorphisms of surfaces, Bull. Amer. Math. Soc. 19 (1988), 417-431. MR0956596 (89k:57023)

[23] _ over the circle, math.GT/9801045.

[24] S. Vidussi: Lagrangian surfaces in a fixed homology class: Existence of knotted Lagrangian tori, J. Differential Geom. (to appear), math.GT/0311174.

Department of Mathematics and Statistics, McMaster University, Hamilton, OnTARIO, CANADA L8S 4K1

Current address: Department of Mathematics, Koç University, Istanbul, 34450, Turkey

E-mail address: tetgu@ku.edu.tr

Department of Pure Mathematics, University of Waterloo, Waterloo, Ontario, CANADA N2L 3G1

E-mail address: dmckinnon@math.uwaterloo.ca

URL: http://www.math. uwaterloo.ca/ dmckinnon/

Department of Pure Mathematics, University of Waterloo, Waterloo, Ontario, CANAdA N2L 3G1

E-mail address: bdpark@math.uwaterloo.ca

URL: http://www.math. uwaterloo.ca/ bdpark/ 